\title{
A EXPERIÊNCIA PIONEIRA DO ENSINO À DISTÂNCIA EM TREINAMENTO DE USUÁRIOS DE BIBLIOTECAS UNIVERSITÁRIAS
}

\author{
(THE PIONEERING EXPERIENCE OF DISTANCE EDUCATION RESEARCH IN THE TRAINING OF \\ UNIVERSITY LIBRARY USERS)
}

\author{
Luciana Pizzani \\ Rosemary Cristina da Silva \\ Denise de Cássia Moreira Zornoff \\ Lucas Frederico Arantes \\ Universidade Estadual Paulista Julio de Mesquita Filho (Brasil)
}

\section{RESUMO}

Esse artigo tem como objetivo relatar a experiência da construção e implantação do primeiro curso à distância sobre normalização de trabalhos científicos e pesquisas em bases de dados como complemento das atividades de educação de usuários em bibliotecas universitárias. O trabalho foi desenvolvido pela Divisão Técnica de Biblioteca e Documentação da Universidade Estadual Paulista Julio de Mesquita Filho-Campus Botucatu-Rubião Junior e o Núcleo de Educação a Distância e Tecnologias da Informação em Saúde da Faculdade de Medicina de Botucatu. Foi realizado de agosto a novembro de 2009, distribuído em 10 aulas. Dos 128 alunos inscritos, $63 \%$ concluíram o curso e $37 \%$ desistiram em diferentes pontos do aprendizado. Verificou-se que a participação dos alunos revela aceitação positiva da metodologia adotada, motivando a sua extensão a toda comunidade acadêmica. A experiência pioneira do trabalho com equipe multidisciplinar situa o bibliotecário como mediador do conhecimento, acompanhando a evolução do processo educativo.

Palavras-chave: educação à distância, biblioteca universitária, capacitação de usuários.

\begin{abstract}
The aim of this article is to relate our experiences of creating and implementing the first distance education course in "Database norms for scientific publications and research: a complement to educational activities for university library users". The research was carried out by the Technical Division of Librarianship and Documentation at Universidade Estadual Paulista "Julio de Mesquita Filho" (Botucatu- Rubiã Junior campus) and the Center for Distance Health Education and Information Technology at the Botucatu Faculty of Medicine. The study was carried out from August to November
\end{abstract}


2009 using 10 different university classes. Out of the 128 enrolled students, $63 \%$ completed their studies. $37 \%$, however, terminated their studies at various stages of the course. Despite these problems, there was positive feedback about adopting the new methodology, which subsequently led to its widespread application in the academic community. The pioneering experience of multidisciplinary teamwork places librarians as "mediators of knowledge", thus enabling them to become part of the development of the educational process.

Keywords: education distance, university libraries, users education.

O mundo atual é marcado por uma série de mudanças tecnológicas que estão afetando o modo de ver, viver, ser e de pensar do homem. Também a educação e o sistema educacional têm sido pressionados a caminhar por novos rumos. Fatores como o acelerado ritmo de vida das pessoas e das tecnologias incorporadas aos equipamentos de informática e de comunicação, a maior exigência de capacitação dos trabalhadores e o acirramento da concorrência no mercado de trabalho têm levado muitas pessoas a buscarem cursos mediados ou a distância como forma de obterem graduação ou aperfeiçoamento profissional. Assim, a prática da Educação a Distância (EaD) transformou-se, no final do século XX, numa das ferramentas utilizadas no âmbito da Educação (Arieira et al., 2009).

Também as Instituições de Ensino Superior passaram a oferecer aos estudantes acesso às tecnologias de informação e comunicação: Internet, ambientes educacionais na Web, videoconferência e teleconferência, suportes hipermídia (CDROM, bancos de dados multimídia interativos online), sistemas de simulação etc. O uso dessas redes de telecomunicação e dos suportes multimídia interativos estão sendo integrados dando outra dimensão aos processos de ensino e aprendizagem. Assim, esses processos se desenvolvem em um ambiente híbrido, ora por situações presenciais ora por situações virtuais (Arieira et al., 2009).

Acompanhando essa evolução em direção ao uso das novas tecnologias e ao ensino à distância a Universidade Estadual Paulista Julio de Mesquita Filho (UNESP), Campus Botucatu Rubião Junior, criou, em setembro de 2001, o Núcleo de Educação à Distância e Tecnologias da Informação em Saúde (Nead.Tis) com o objetivo de promover a implantação e divulgação de novas tecnologias em EaD na Faculdade de Medicina de Botucatu (FMB).

A Divisão Técnica de Biblioteca e Documentação, percebendo a oportunidade de aliar o treinamento de usuários ao trabalho desenvolvido pelo Nead.Tis, elaborou um curso de extensão universitária sobre normalização de trabalhos científicos e pesquisa em bases de dados para capacitação de seus usuários. 
Esse artigo tem como objetivo relatar a primeira oportunidade da Divisão Técnica de Biblioteca e Documentação no treinamento de usuários na modalidade $\mathrm{EaD}$, utilizando como metodologia de trabalho a ferramenta Moodle.

O curso de extensão recebeu o nome de Orientação à elaboração de trabalhos científicos e pesquisa em bases de dados, com carga horária de 6ohs, ministrado no período de agosto a novembro de 2009.

Apresentou como objetivo geral oferecer uma visão sistêmica aos alunos de graduação, pós-graduação, docentes e demais interessados acerca dos princípios teóricos-conceituais sobre a elaboração de trabalhos científicos.

A realização do curso justificou-se pela crescente demanda de treinamentos solicitados aos bibliotecários de referência pelos docentes dos cursos de graduação e Pós-Graduação da Faculdade de Medicina de Botucatu, Instituto de Biociências de Botucatu e Faculdade de Medicina Veterinária e Zootecnia, da Universidade Estadual Paulista - Campus Botucatu-Rubião Junior.

\section{CONHECENDO A UNIVERSIDADE ESTADUAL PAULISTA JÚLIO DE MESQUITA FILHO - UNESP ${ }^{1}$}

A UNESP (Universidade Estadual Paulista "Júlio de Mesquita Filho") é uma das maiores e mais importantes universidades brasileiras, com destacada atuação no ensino, na pesquisa e na extensão de serviços à comunidade.

Mantida pelo Governo do Estado de São Paulo, é uma das três universidades públicas de ensino gratuito, ao lado da USP (Universidade de São Paulo) e da Unicamp (Universidade Estadual de Campinas).

Há, no entanto, uma peculiaridade que a distingue das demais: é a única universidade presente em praticamente todo o território paulista. Sua estrutura multicâmpus está presente em 23 cidades do Estado de São Paulo, sendo 21 no Interior; um na Capital do Estado, São Paulo; e um em São Vicente - o primeiro de uma universidade pública no Litoral Paulista.

Criada em 1976, a partir de institutos isolados de ensino superior que existiam em várias regiões do Estado de São Paulo, a UNESP tem 32 unidades, que oferecem 169 opções de cursos de graduação em 64 profissões de nível superior, que formam, por ano, 6 mil novos profissionais e são responsáveis por 115 programas de Pós- 
Graduaçào, que oferecem 110 mestrados acadêmicos, 4 mestrados profissionais e 91 doutorados acadêmicos.

A infraestrutura da Universidade inclui 1.900 laboratórios e 30 bibliotecas, com 900 mil livros. Além disso, há, à disposição de alunos e professores, museus, hortos, biotérios, jardins botânicos e cinco fazendas experimentais, perfazendo uma área total de 62,8 milhões de $\mathrm{m}^{2}$, sendo 745,4 $\mathrm{mil} \mathrm{m}^{2}$ de área construída.

\section{A FACULDADE DE MEDICINA DE BOTUCATU (FMB) E O NEAD.TIS ${ }^{2}$}

Referência brasileira e internacional em ensino, pesquisa e extensão, a Faculdade de Medicina de Botucatu oferece cursos de graduação em Medicina Humana e Enfermagem, 36 programas de residência médica, 53 de aprimoramento profissional, 8 de pós-graduação e 2 de mestrado profissionalizante. Na graduação, $o$ curso de Medicina Humana oferece 90 vagas e o de Enfermagem 30. Juntos reúnem 284 docentes e 1.475 servidores.

Implantada em 1963 como Faculdade de Ciências Médicas e Biológicas de Botucatu (FCMBB) e incorporada à Unesp em 1976, seus cursos possuem uma sólida base científica, postura ética e uma forte visão humanística e de comprometimento com a cidadania. Seu objetivo é formar profissionais com senso crítico, conscientes de seu papel na sociedade.

Em setembro de 2001, a Faculdade de Medicina de Botucatu criou o Núcleo de Educação à Distância e Tecnologias da Informação em Saúde (Nead.Tis) que tem como objetivos: promover a divulgação de novas tecnologias em EAD; promover a educação continuada em EAD como parte do ferramental pedagógico para os docentes da FMB; assessorar e avaliar projetos e cursos em EAD na FMB nos seus vários níveis: planejamento, design, produção, aplicação, gerenciamento, avaliação e monitoramento de frequência e desempenho; disponibilizarão dos conteúdos de mídia na FMB, de acordo com os princípios ditados pela UNESP.

É um órgão ligado diretamente à Comissão Permanente de Extensão Universitária (CPEU) da FMB e à Pró-Reitoria de Extensão Universitária da UNESP (PROEX).

Assim, no ano de 2001, foi criada a Escola Médica Virtual como um ambiente de ensino a distância. 


\section{A DIVISÃo TÉCNICA DE BIBLIOTECA E DOCUMENTAÇÃO ${ }^{3}$}

A Divisão Técnica de Biblioteca e Documentação abriga um acervo e oferece serviços de informação de apoio ás atividades de ensino, pesquisa e extensão desenvolvidas pelas Unidades do Campus de Botucatu: Faculdade de Medicina de Botucatu, Faculdade de Medicina Veterinária e Zootecnia e Instituto de Biociências de Botucatu. O acervo é, especialmente voltado para as áreas de graduação em Medicina, Enfermagem, Medicina Veterinária, Zootecnia, Biologia, Nutrição e Física Médica; programas de pós-graduação nas áreas de Medicina Humana, Medicina Veterinária e Biologia, residências em Medicina e Medicina Veterinária, e cursos de aprimoramento e especialização ofertados pelas Unidades locais.

Foi criada em 1964 com a missão de contribuir ao desenvolvimento integral da sociedade, promovendo o acesso e a produção do conhecimento, nas Unidades do Campus de Botucatu e Unesp, prioritariamente, e também a todo cidadão, por meio de atendimento direto, ou por intermédio da participação de sistemas locais, regionais e internacionais de informação.

Até dezembro de 2010 possui 4821 usuários inscritos distribuídos nas seguintes categorias: alunos de graduação: 2.534; alunos de pós-graduação: 597; docentes: 479; funcionários: 670; externos: 13; alunos rede Unesp : 10; alunos de especialização: 74; estagiários: 99; residentes: 241; aposentados : 7; alunos de aprimoramento:97.

Oferece vários serviços, entre eles: alimentação de bases de dados, assessoria de publicações, comutação, empréstimo entre bibliotecas, fotocópias, cursos e treinamentos sobre pesquisas em bases de dados e normalização de trabalhos científicos.

Desde o início das suas atividades a Divisão Técnica de Biblioteca e Documentação contatou a necessidade de capacitar os usuários na utilização dos recursos informacionais, iniciando-se os treinamentos formais com o objetivo de habilitar os usuários na utilização dos serviços oferecidos à comunidade acadêmica. Com a introdução do ensino a distância os treinamentos passaram a ser ministrados de forma híbrida, tanto presencial quando à distância.

\section{A EDUCAÇÃo A DISTÂNCIA (EAD)}

Para Garcia e Baptista (2007) o avanço das tecnologias de comunicação e informação da sociedade suscita transformações nas relações sociais e culturais, 
repercutindo nas diversas formas do agir humano, desde a construção do saber até as relações entre ciência, educação, sociedade e trabalho. As novas alternativas de promoção da educação que surgem nesse contexto ampliam as possibilidades metodológicas e organizacionais e disponibilizam diversos ambientes para fins didáticos, de capacitação e formação, inclusive em nível de graduação e pósgraduação. A educação a distância (EaD), por exemplo, ganha novas dimensões com as tecnologias digitais, que ampliam as possibilidades de capilarização dos processos de formação e disseminação de informações e conhecimentos, disponibilizando ambientes virtuais e interativos de aprendizagem que colocam, sem dúvida, novos desafios e alternativas para se pensar os processos formativos e de capacitação.

A incorporação de tecnologias pelos cursos oferecidos nas universidades, na medida em que essas tecnologias iam se tornando disponíveis, foi abrindo espaços e criando oportunidades para serviços típicos de apoio bibliotecário, entre eles, a educação a distância, podendo ser considerada como uma oportunidade para ampliarem o papel do bibliotecário na capacitação de usuários. Assim as bibliotecas buscam cumprir seu papel de educadora, apoiando as atividades da universidade e centrando suas atividades nas necessidades informacionais de seus usuários (Ronchesel y Pacheco, 2008).

O termo educação a distância possui diversas definições, mas no contexto deste trabalho adotaremos a definição de Moran (2002). O autor a define como o processo de ensino-aprendizagem, mediado por tecnologias, onde professores e alunos estão separados espacialmente, mas podem estar conectados ou interligados por tecnologias, como a Internet.

Faria (2002), faz um resumo sobre a criação do ensino a distância no Brasil desde o ensino por correspondência até o surgimento da Internet. Segundo a autora, a educação a distância surgiu no Brasil como modalidade de ensino no início do século XIX, a partir da necessidade de atualizar conhecimentos e preparar pessoas impossibilitadas de cursar o ensino regular ou profissionalizante.

Até o início do século XX os programas educacionais imprimiam os materiais e os enviava via correios, por isso ficaram conhecidos como "ensino por correspondência". Um pouco mais adiante surge o rádio e a EaD passou a ser desenvolvida também por meio de programas transmitidos por emissoras de rádio em horários específicos. $\mathrm{Na}$ década de 1960 surgiu a televisão e os programas da Fundação Educacional e Cultural Padre Anchieta; em 1969 a TV Cultura, juntamente com a Fundação Padre Anchieta desenvolveu o Projeto Minerva; no final dos anos de 1970, a Rede Globo lançou o 
Telecurso $2^{\circ}$ Grau com grande sucesso que gerou o Telecurso $1^{\circ}$ grau e o Telecurso 2000 sendo utilizado em larga escala por empresas como a Petrobrás, Fundação Roquete Pinto, SEBRAE, SENAC, SENAT, SENAR e SENAI para capacitação dos seus funcionários (Faria, 2002).

No ensino superior a EAD teve início na década de 1990 facilitando as atividades educacionais de alunos que não poderiam se deslocar de suas residências/cidades por muito tempo e também para cursos de extensão universitária. As pioneiras foram a Universidade de Brasília (UnB) e a Universidade Federal de Santa Catarina (UFSC) (Faria, 2002).

Com a consolidação do ensino a distância, em 1995, foi criada a Secretaria de Educação a Distância (SEED), pelo MEC, com o objetivo de levar para a escola pública toda a contribuição que os métodos, técnicas e tecnologias de educação a distância podem prestar à construção de um novo paradigma para a educação brasileira (Faria, 2002).

Tendo em vista tal contexto, é criado em 2001 o Núcleo de Educação a Distância e Tecnologias da Informação em Saúde (Nead.Tis), pela Faculdade de Medicina de Botucatu, com o objetivo de promover a implantação, divulgação e educação continuada em EaD como parte do ferramental pedagógico para os docentes da FMB.

A Divisão Técnica de Biblioteca e Documentação percebeu a possibilidade de estabelecer uma parceria com o Nead.Tis para proporcionar treinamento aos usuários referente à normalização de trabalhos científicos e pesquisas em bases de dados.

Esse tipo de treinamento é bastante solicitado por parte dos docentes da Faculdade de Medicina de Botucatu, Instituto de Biociências e Faculdade de Medicina Veterinária e Zootecnia do campus da Unesp-Rubião Junior. Em dados coletados no Relatório de Atividades realizado anualmente para a Coordenadoria Geral de Bibliotecas, consta que no ano de 2009 foram realizados 17 treinamentos para os alunos da graduação, pós-graduação e profissionais da área médica, totalizando 475 usuários, o que equivale a aproximadamente mais de um curso por mês.

Essa amostra vem reiterar a motivação para implementar essa prática frequente em nosso sistema de informação, com o objetivo de atender a demanda e atingir mais usuários da rede Unesp e potenciais interessados, otimizando o tempo. 
A ferramenta de aprendizagem utilizada foi o Moodle (Modular Object Oriented Distance Learning), desenvolvido em 1999 pelo australiano Martin Dougiamas. O ambiente Moodle pode ser definido como um conjunto de ferramentas computacionais (fórum, diário, tarefa com envio de um arquivo, lição e Chat) que apoiam a execução de um curso à distância. É considerado um software livre, gratuito, que pode ser baixado, utilizado e modificado por qualquer indivíduo em todo o mundo (Brito y Alves, 2005).

Desde a década de 1980 os programas de educação de usuários baseiam-se no pressuposto de que as pessoas necessitam de informações armazenadas e organizadas em bibliotecas segundo técnicas complexas e até sofisticadas, o que dificulta que o leitor seja bem-sucedido em suas tentativas de encontrar o material que necessita. É na biblioteca universitária que esses programas têm-se desenvolvido de forma mais consciente, por serem os estudantes seus frequentadores regulares, por um período de tempo bastante longo (Campos, Magalhães, 1982).

Com a evolução das tecnologias da informação, o papel do bibliotecário passou por profundas transformações para acompanhar as necessidades dos usuários; passou a ser o de educador (trainer), aquele que capacita os usuários a se tornarem permanentemente autônomos tanto na busca e recuperação da informação quanto na realização de seus trabalhos acadêmicos (Cuenca, 1999).

Ainda nessa perspectiva, conforme afirma Dudiziak (2007) foi na aplicação do ensino a distância como uma ferramenta aliada a área da Ciência da Informação, sua interdisciplinaridade, que o profissional bibliotecário, assumiu o papel de mediador pedagógico, tornando-se educador, pois passou a organizar programas decompetência informacional em conjunto com professores e gestores, ministrando aulas em diversos espaços, executando projetos informacionais com foco na educação voltada para a competência em informação (information literacy education), observando a importância do acolhimento e do aprendizado significativo, aprimorando seus conhecimentos educacionais e pedagógicos.

\section{MATERIAIS E MÉTODOS}

A implantação do curso Orientação à elaboração de trabalhos científicos e pesquisa em bases de dados foi desenvolvida em quatro etapas: 
Etapa 1: Contato com a direção do Nead.Tis para verificar a viabilidade do curso. O grupo demonstrou interesse em participar do projeto colaborando com a tutoria virtual, apoio tecnológico e burocrático para a sua implantação.

Etapa 2: Elaboração da proposta contendo: o plano de ensino; o programa compreendendo as atividades práticas para avaliação dos alunos; o sistema de avaliação.

O programa compreendeu 10 aulas, distribuídas na seguinte sequência:

- Aula 1: Apresentação do curso;

- Aula 2: ABNT 14724;

- Aula 3: ABNT 6023;

- Aula 4: Elaboração de referências segundo as normas de Vancouver;

- Aula 5: ABNT 6028;

- Aula 6: ABNT: 10520;

- Aula 7: Técnicas para elaboração de revisão de literatura;

- Aula 8: Levantamento bibliográfico em bases de dados com acesso livre;

- Aula 9: Levantamento bibliográfico em bases de dados com acesso restrito;

- Aula 10: Elaboração de um projeto de pesquisa como Trabalho de Conclusão de Curso (TCC).

Cada aula foi composta por quatro atividades destinadas a fixação do conteúdo:

- Atividade 1: estudo do material postado pelos professores;

- Atividade 2: leitura de textos científicos relacionados ao conteúdo de cada aula;

- Atividade 3: questionário com perguntas fechadas para avaliação do aluno;

- Atividade 4: atividades lúdicas (palavras-cruzadas, jogos de frases desordenadas, jogo de ligação).

Com relação às atividades lúdicas Leite (2005) acredita na importância e no auxílio do lúdico como estratégia do professor que busca trabalhar conteúdo, de forma prazerosa e eficaz com seus discentes. O ser humano (em especial, o adulto) aprende mais e melhor quando é exposto a diferentes estímulos no processo de assimilação de conhecimento. 
Os critérios de avaliação foram estabelecidos da seguinte maneira:

- Realização das tarefas das aulas;

- Participação no fórum de atividades;

- Participação nos chats;

- Trabalho de Conclusão de Curso (TCC): elaboração de um projeto de pesquisa com tema de interesse do aluno.

As notas tiveram os seguintes critérios:

- Não realizou: zero;

- Deficiente: 1-4 (não atendeu ao objetivo proposto no exercício);

- Regular: 5-6 (atendeu parcialmente ao objetivo proposto no exercício);

- Adequado: 7-9 (atendeu ao objetivo proposto no exercício);

- Excelente: 10 (superou ao objetivo proposto no exercício).

O TCC seguiu um roteiro específico de avaliação com o objetivo de avaliar todo o aprendizado do aluno (utilização das normas quanto à estrutura do trabalho científico, normalização de referências, palavras-chave, formatação, linha de raciocínio e embasamento teórico do projeto).

Etapa 3: Encaminhamento pelo Nead.Tis de toda a documentação referente à proposta do curso para regularização junto à Pró-Reitoria de Extensão (ProEx), da Faculdade de Medicina de Botucatu.

Etapa 4: Divulgação através de e-mail enviado pelo Nead.Tis a todos os alunos, ex-alunos, docentes e funcionários da Faculdade de Medicina de Botucatu, público alvo escolhido para a primeira experiência de um curso totalmente a distância.

As atividades do curso tiveram início no mês de agosto do ano 2009. A partir dessa data, as aulas eram disponibilizadas semanalmente (às segundas-feiras) no ambiente de aprendizagem Moodle, sendo que os alunos tinham o período de uma semana para a realização de todas as atividades. As aulas encerraram-se no final do mês de outubro e após essa data foi estabelecido o prazo de um mês e meio para a entrega do Trabalho de Conclusão de Curso, encerrando-se em dezembro de 2009. 
O curso contou com uma equipe de 8 profissionais: duas bibliotecárias da Divisão Técnica de Biblioteca e Documentação - Campus Botucatu Rubião Junior, eram as professoras e responsáveis pela elaboração do conteúdo das aulas e seis tutores virtuais foram encarregados de dar suporte técnico referente ao uso da plataforma Moodle e também incentivar e motivar os alunos durante o curso.

Cada tutor ficou responsável por um grupo de 20 alunos em média, para facilitar a dinâmica do curso.

Segundo Emerenciano et al. (2007) o tutor é um elemento chave para o desenvolvimento cognitivo do estudante nas atividades individuais e coletivas ao longo da disciplina. Ele é responsável por acompanhar, orientar, estimular e provocar o estudante a construir o seu próprio saber, desenvolver processos reflexivos e criar um pronunciamento marcadamente pessoal.

Ainda com relação a equipe de trabalho Leite (2005) afirma a necessidade de uma equipe interdisciplinar para a construção de conteúdos virtuais. Defende a ideia que uma equipe deve ter o perfil generalista-especialista, e habilidades em Teorias da Aprendizagem, Tecnologia Educacional e conhecimento específico do conteúdo a ser trabalhado.

Esses fatores podem ser observados claramente na formação da equipe multidisciplinar composta por 2 bibliotecários, 6 tutores virtuais sendo 1 docente da Faculdade de Medicina e também coordenador do Nead.Tis e 5 profissionais ligados à área de informática.

\section{RESULTADOS}

Em um primeiro momento contamos com 188 inscrições. Após um período de uma semana após o encerramento das inscrições foi solicitada a confirmação da matrícula e interesse pelo curso onde 128 alunos confirmaram a participação.

\begin{tabular}{|l|c|c|}
\hline \multicolumn{1}{|c|}{ Inscrições } & Frequência & $\%$ \\
\hline $\begin{array}{l}\text { Profissionais da área médica (Médicos, Enfermeiros, Farmacêuticos, } \\
\text { Psicólogos, Fisioterapeutas, Fonoaudiólogos, Assistente Social, entre outros) }\end{array}$ & 44 & 34,38 \\
\hline Funcionários (Assistente administrativo, Administrador, Analista de Sistemas) & 26 & 20,31 \\
\hline Alunos de Graduação & 23 & 17,98 \\
\hline Alunos de Pós-graduação & 19 & 14,84 \\
\hline
\end{tabular}




\begin{tabular}{|l|c|c|}
\hline \multicolumn{1}{|c|}{ Inscrições } & Frequência & $\%$ \\
\hline Docentes & 12 & 9,37 \\
\hline $\begin{array}{l}\text { Demais interessados (Professor rede pública de ensino, Produtor cultural, } \\
\text { Educador Físico) }\end{array}$ & 04 & 3,12 \\
\hline Total & 128 & 100 \\
\hline
\end{tabular}

Tabela 1. Participantes do curso

Na Tabela 1 podemos verificar que o curso despertou interesse dos alunos de graduação, pós-graduação, docentes e funcionários, conseguindo contemplar toda a comunidade acadêmica da Faculdade de Medicina de Botucatu.

Desse total, verificou-se que 80 (63\%) alunos concluíram o curso e 48 (37\%) desistiram em diferentes pontos do aprendizado.

Com relação às notas, constata-se que houve um ótimo aproveitamento do curso: 54 alunos obtiveram média final entre 90,00 a 100,00 pontos, 23 alunos com média entre 80,00 a 89,00 e três alunos com média entre 70,00 a 79,00 pontos.

Confrontando os dados dos usuários inscritos no curso a distância e o Relatório de Atividades junto à Coordenadoria Geral de Bibliotecas dos cursos presenciais ministrados no ano de 2009, destacamos a grande ocorrência e também o interesse das diversas categorias de usuários nos treinamentos realizados na temática. A Tabela 2 ilustra o número de usuários que passaram por treinamento presencial em normalização e pesquisas em bases de dados.

\begin{tabular}{|l|c|c|c|}
\hline Treinamentos presenciais ministrados no ano de 2009 & Curso & Usuários & $\%$ \\
\hline Cursos de Graduação & 3 & 129 & 27,15 \\
\hline Cursos de Pós-graduação & 8 & 171 & 36,00 \\
\hline Profissionais da área médica & 4 & 155 & 32,63 \\
\hline Demais interessados & 2 & 20 & 4,22 \\
\hline Total & 17 & 475 & 100,00 \\
\hline
\end{tabular}

Tabela 2. Treinamentos presenciais ministrados no ano de 2009 referentes à temática normalização

Os resultados aqui apresentados merecem uma interpretação que ressalte a importância e eficiência em aliar o ensino à distância a prática bibliotecária de capacitação de usuários. 
Se observarmos que no ano de 2009 foram realizados 17 treinamentos para capacitar 475 usuários, o mesmo treinamento realizado a distância com duração de três meses capacitou 80 usuários.

Esse resultado se multiplicar por um ano, seriam realizados quatro treinamentos à distância para capacitar 316 usuários (utilizando como base o total de usuários que concluíram o curso que foi de 80).

Em relação ao treinamento presencial, onde foram necessários 17 treinamentos para atender uma população de 475 usuários, levando em conta o deslocamento do profissional bibliotecário, deslocamento dos usuários e tempo destinado a essas atividades, fica evidente a questão custo-benefício dessa iniciativa.

Além de poder atender mais usuários com menor número de treinamentos, o profissional bibliotecário bem como os usuários poderão utilizar o tempo que era destinado ao treinamento presencial para se dedicar a outras atividades, já que no ensino a distância permite ao participante adequar de forma mais flexível o seu tempo de estudo.

\section{CONSIDERAÇÕES FINAIS}

O surgimento de novas tecnologias nas áreas de informação e comunicação alterou o panorama das bibliotecas universitárias nas últimas décadas exigindo mudanças tanto do profissional da informação, quanto do desempenho da biblioteca universitária no que se refere à prestação de serviços aos usuários. Novos mecanismos de trabalho foram introduzidos nas bibliotecas universitárias exigindo tanto do profissional quanto dos usuários atualização constante para a produção do conhecimento.

A experiência inovadora e ao mesmo tempo desafiadora em implantar um curso a distância reflete perfeitamente essa nova realidade do fazer biblioteconômico frente à capacitação de usuários, requerendo atualização constante com relação às novas tecnologias e metodologias aplicadas aos treinamentos dos usuários de bibliotecas universitárias.

Podemos verificar com a grande participação e empenho dos alunos que a capacitação e treinamento de usuários utilizando a metodologia da educação a distância foi realizado com sucesso pela Divisão Técnica de Biblioteca e Documentação e Nead.Tis. 
Outro fator importante é relatar que a experiência do trabalho com equipe multidisciplinar revela as importantes contribuições de cada área situando a biblioteca e o bibliotecário como mediadores do conhecimento, acompanhando a evolução do processo educativo.

O sucesso do curso também motiva a extensão do mesmo e a criação de outros para a comunidade acadêmica do campus Botucatu-Rubião Junior e, em um futuro próximo, até mesmo para as demais unidades da Universidade Estadual Paulista Julio de Mesquita espalhados pelo interior do Estado de São Paulo mediante capacitação de mais bibliotecários interessados em percorrer os novos caminhos que a educação a distância permite trilhar.

Esse fato situa a experiência a educação à distância como uma das formas de mediação pedagógica, contribuindo amplamente nos processos de ensino e aprendizagem.

\section{NOTAS}

1 Universidade Estadual Paulista (2011). Histórico da criação da UNESP. Botucatu: UNESP. [en línea] Disponível em: www.unesp.br (consulta 2011, 13 de março).

2 Universidade Estadual Paulista (2011). Apresentação. Botucatu: UNESP. Disponível em: http://www.fmb.unesp.br/instituicao/apresentacao.php (consulta 2011, 13 de março).

3 Universidade Estadual Paulista. Divisão Técnica de Biblioteca e Documentação (2011). Apresentação. Botucatu: UNESP. [en línea] Disponível em: http://www. biblioteca.btu.unesp.br/instituicao/apresentacao.php (consulta 2011, 13 de março).

\section{REFERÊNCIAS BIBLIOGRÁFICAS}

Arieira, J. O.; Dias-Arieira, C. R.; Sacomano, J. B.; Bettega, M. O. P. (2009). Avaliação do aprendizado via educação a distância: a visão dos discentes. Ensaio Avaliação e Políticas Públicas em Educação, 17 (63), (313-340).

Brito, M. S. S.; Alves, L. R. (2005). O ambiente Moodle como apoio ao ensino presencial. Anais do XII Congresso Internacional de Educação a Distancia. Florianópolis.
Cuenca, A. M. B. (1999). O usuário final da busca informatizada: avaliação da capacitação no acesso a bases de dados em biblioteca acadêmica. Ciência da Informação, 28, (3), (291-299).

Dudziak, E. A. (2007). O bibliotecário como agente de transformação em uma sociedade complexa: integração entre ciência, tecnologia, desenvolvimento e inclusão social. PontodeAcesso, 1 (1), (88-98). [en línea] Disponível em: 
http://www.portalseer.ufba.br/index. $\mathrm{php} /$ revistaici/article/view/1396/878 (consulta 2011, 13 de março).

Emerenciano, M. S. J.; Sousa, C. A. L.; Freitas, L. G. (2007). Ser presença como educador, professor e tutor. [en línea] Disponível em: http://www.abed.org.br (consulta 2011, 13 de março).

Faria, E.T.(2002). Interatividadee mediação pedagógica na educação a distância. 214f. Tese (Doutorado) - Faculdade de Educação, Pontifícia Universidade Católica do Rio Grande do Sul, Porto Alegre.

Garcia, R. M.; Baptista, R. (2007). Educação à distância para a qualificação dos profissionais do SUS: perspectivas e desafios. Revista Baiana de Saúde Pública, 31 (supl.1), (70-78).
Leite, L. O. (2005). O lúdico na educação à distância. Novas Tecnologias na Educação, 3 (1), (1-8).

Magalhães, C. M.; Campos, M. H. A. (1982). Treinamento de usuários de biblioteca universitária: o curso na Universidade Federal de Minas Gerais. Revista de Biblioteconomia de Brasília, 10 (2), (123-128).

Moran, J. M. (2002). O que é educação à distância. São Paulo. ECA.

Ronchesel, M. H. S.; Pacheco, L. K. (2008). Diretrizes para cursos a distância de capacitação de usuários em bibliotecas universitárias. Revista Brasileira de Biblioteconomia e Documentação, 4, (2), (233-243).

\section{PERFIL ACADÊMICO E PROFISSIONAL DOS AUTORES}

Luciana Pizzani. Bibliotecária, Universidade Estadual Paulista Julio de Mesquita Filho, Divisão Técnica de Biblioteca e Documentação, Seção Técnica de Referência e Atendimento ao Usuário e Documentação, Botucatu, SP, Brasil. Doutoranda do Programa de Pós-Graduação em Educação Especial da Universidade Federal de São Carlos (UFSCar)

E-mail: luciana@btu.unesp.br

Rosemary Cristina da Silva Bibliotecária, Universidade Estadual Paulista Julio de Mesquita Filho, Divisão Técnica de Biblioteca e Documentação, Seção Técnica de Referência e Atendimento ao Usuário e Documentação, Botucatu, SP, Brasil. Doutoranda do Programa de Pós-Graduação em Educação - Universidade Federal de São Carlos (UFSCar).

E-mail: meire@btu.unesp.br

Denise de Cássia Moreira Zornoff Professora Doutora da Faculdade de Medicina de Botucatu. Coordenadora do Nead.Tis, Universidade Estadual Paulista Julio de Mesquita Filho, Faculdade de Medicina, Botucatu, SP, Brasil.

E-mail: dcmzornoff@fmb.unesp.br 
Lucas Frederico Arantes. Nead.Tis, Universidade Estadual Paulista Julio de Mesquita Filho, Faculdade de Medicina, Botucatu, SP, Brasil.

E-mail: lfarantes@fmb.unesp.br

ENDEREÇO DOS AUTORES:

Divisão Técnica de Biblioteca e Documentação

Distrito de Rubião Junior, $\mathrm{s} / \mathrm{n}^{\circ}$, Botucatu, São Paulo, Brasil

CEP: 18.618-970, Caixa Postal: 502

Fecha de recepción del artículo: 21/03/11

Fecha de aceptación del artículo: 26/09/11

\section{Como citar este artículo:}

Pizzani, L.; da Silva, R. C.; de Cássia, D.; Zornoff, M.; Arantes, L. F. (2012). A experiência pioneira do ensino à distância em treinamento de usuários de bibliotecas universitárias. RIED. Revista Iberoamericana de Educación a Distancia, volumen 15, $\mathrm{n}^{\circ}$ 1, pp. 143-158. 